\title{
Poster: Characterizing Uncertainties of Wireless Channels in Connected Vehicles
}

\author{
Elahe Soltanaghaei* \\ University of Virginia \\ Charlottesville, VA \\ es3ce@virginia.edu
}

\author{
Mahmoud Elnaggar* \\ University of Virginia \\ Charlottesville, VA \\ mae3tb@virginia.edu
}

\author{
Katie Kleeman \\ University of Virginia \\ Charlottesville, VA \\ knk9ab@virginia.edu
}

\author{
Kamin Whitehouse \\ Amazon \& University of Virginia \\ Charlottesville, VA \\ whitehouse@virginia.edu
}

\author{
Cody Fleming \\ University of Virginia \\ Charlottesville, VA \\ fleming@virginia.edu
}

\begin{abstract}
The performance of autonomous cars can be greatly enhanced through wireless coordination. However, mobility has traditionally been a challenge for wireless networks due to rapid fluctuation of the signal quality. Current control systems handle this challenge by slowing down the vehicle to preserve safety. However, in this research, we demonstrate that we can robustly characterize the channel quality by mapping the multipath signals to the dynamics of the physical environment, thus controlling the trajectory of the mobile agent to a safe efficient motion path. This allows mobile systems to realize the performance benefits of wireless coordination while providing safety.
\end{abstract}

\section{KEYWORDS}

Wireless, CSI, multipath propagation, connected vehicles, channel ACM Reference format:

Elahe Soltanaghaei, Mahmoud Elnaggar, Katie Kleeman, Kamin Whitehouse, and Cody Fleming. 2019. Poster: Characterizing Uncertainties of Wireless Channels in Connected Vehicles. In Proceedings of The 25th Annual International Conference on Mobile Computing and Networking, Los Cabos, Mexico, October 21-25, 2019 (MobiCom '19), 3 pages.

https://doi.org/10.1145/3300061.3343409

\section{INTRODUCTION}

Mobile systems has gain significant traction in the marketplace and are expected to transform industries such as driving, consumer robotics, and aerial drones. As the density of these autonomous systems increases, their performance and capabilities will be greatly enhanced through wireless coordination. For example, vehicle platoons increase highway throughput and fuel efficiency by using a wireless coupling to brake and accelerate simultaneously; or vehicles move around blind corners at high speed by leveraging the sensing capabilities of the cars ahead of them. However, mobility

\section{${ }^{*}$ Co-primary Authors}

Permission to make digital or hard copies of part or all of this work for personal or classroom use is granted without fee provided that copies are not made or distributed for profit or commercial advantage and that copies bear this notice and the full citation on the first page. Copyrights for third-party components of this work must be honored. For all other uses, contact the owner/author(s).

MobiCom '19, October 21-25, 2019, Los Cabos, Mexico

(c) 2019 Copyright held by the owner/author(s).

ACM ISBN 978-1-4503-6169-9/19/10.

https://doi.org/10.1145/3300061.3343409 has traditionally been a challenge for wireless networks due to rapid fluctuation of the signal quality. Wireless signals are transmitted through unguided media and are therefore greatly affected by the surrounding environment, causing a non-stationary effect on the channel.

The key challenge is that the instability of the wireless channel limits the application of wireless communication in connected vehicles as control systems require to preserve safety while dealing with non-stationary channels. For example, state-of-the-art control systems slow down the vehicle when the wireless connectivity is unstable to preserve safety. There have been many recent development for the formal analysis of control and communication schemes for networked multi-agent mobile systems, however, they assume either a perfect V2V communication [4] or a stationary communication channel [2]. On the other hand, the available channel quality prediction methods purely rely on the network physical and link layer metrics $[1,6]$ and ignore the predictive capability of control systems in defining their own motion through the environment.

In this research, we aim to fill this gap between the communication and control systems by proposing a new channel prediction method that is robust to the dynamics of wireless channels and surrounding environment. The main intuition behind our proposed method is that the variations of the wireless channel are measurable by characterizing multipath propagation and mapping it to the physical environment. In urban environments, wireless signals propagate along multiple paths, reflecting off of surfaces, causing potential destructive interference and loss of packets (shown in Figure 1). To characterize multipath propagation, we leverage the

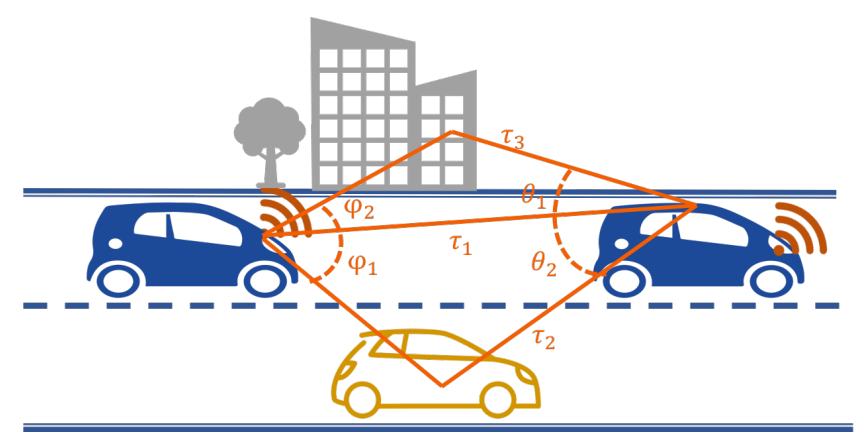

Figure 1: Multipath propagation in urban environments is a main source of wireless channel fluctuations. 

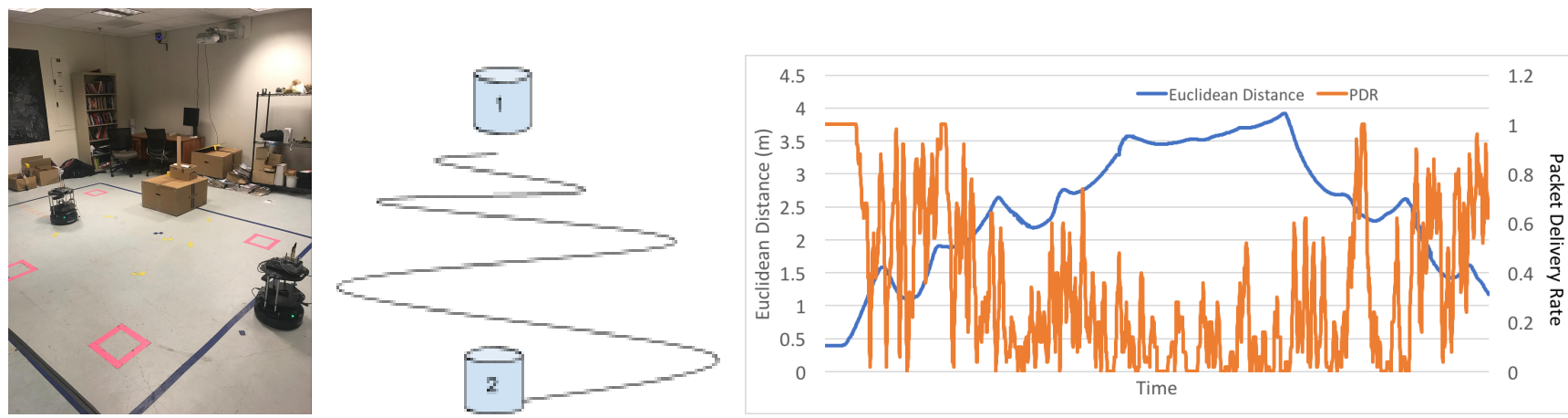

Figure 2: Mobility results in temporal variations of packet delivery rate, which is also a function of the distance between wireless nodes.

physical layer Channel State Information (CSI) measured across multiple antennas and multiple frequencies and map it to Packet Delivery Rate (PDR) as a software metric indicating the future link quality. We demonstrate that the continuity of the car motion and physical environment enables link quality prediction by extracting temporal and spatial characteristics of the channel from short-term and long-term historical data.

To implement this method, we use a Long Short-term Memory (LSTM) network that captures a sequence of past and current channel measurements with a partial model of the physical environment from the future trajectory of the vehicle. We evaluate the system using two turtle bots that are equipped with off-the-shelf wireless radios and $802.11 \mathrm{n}$ protocol for collecting CSI data. The preliminary results show that our proposed approach is robust to fast fluctuations of the channel quality and can predict furture PDR with $91 \%$ accuracy.

\section{METHODOLOGY}

In this paper, we consider a leading and a following vehicle as the two components of a system of vehicles in a platoon. Each vehicle computes it's own motion plan based on the data received from the communications with the preceding vehicle. However, the rapid fluctuations of the wireless channel due to mobility and change of surroundings environments can cause loss of packets and respectively loss of communication between the two vehicles. For the following vehicle to plan its trajectory in these situations, it requires a robust estimation of the channel quality over a future time horizon $T$. In this paper, we consider Packet Delivery Rate (PDR) as a channel quality metric defined by the number of received packets from the total transmitted packets.

To predict PDR over a future time horizon, we model the spatiotemporal factors affecting the wireless channel condition. The dominant source of attenuation in a wireless medium is the diffusion of energy through the environment known as path loss, which is captured by the Friss transmission equation

$$
P_{r} \propto \frac{P_{t}}{d^{n}}
$$

where $\mathrm{d}$ is the distance between transmitter and receiver, $p_{r}$ is the received signal power, $p_{t}$ is transmit signal power, and $n$ is the path loss exponent. As shown in Figure 2, We can clearly see this effect through an empirical experiment with two turtle bots equipped with wireless radios, node 1 is static and node 2 moves to the right and left and gradually moves away from the static node in a snaking motion. As the distance between the two nodes increases, it affects the signal strength and therefore the decrease of PDR. We can also see the temporal variations of the packet delivery rate as the node moves to the right and left.

In addition, the distortions caused by multipath propagation and changes in surrounding environment, caused by mobility, result in a time-varying signal fading. Since the received signal is the superposition of all reflected paths, the destructive interference of multipath signals causes deep fading in the channel response and therefore loss of information. We model the constructive and destructive interference of multipath signals using Channel State Information (CSI) captured from multiple transmitting antennas, multiple receiving antennas, and different frequency subcarriers. In other words, The CSI from the $i^{t h}$ transmit antenna to the $j^{t h}$ receive antenna at the $k^{t h}$ frequency channel is

$$
H_{i j k}=\sum_{n=1}^{N} \alpha_{n} e^{-j 2 \pi \tau_{i j n} f_{k}}
$$

where $\tau_{i j n}$ is the time delay (known as Time of Flight (ToF)) between the $i^{t h}$ transmit and the $j^{t h}$ receive antenna along the $n t h$ path, $f_{k}$ is the frequency of the $k^{t h}$ subchannel, and $\alpha_{n}$ denotes the complex attenuation. The wireless channel quality may alter from good to bad with a small change in path lengths or frequency of the signal, and as can be seen in the equation 4 , the phase component of CSI varies over both frequency and space, thus providing a fine-grained measurement of this effect. We use this feature to characterize multipath signals based on their physical properties such as Angle of Arrival (AoA), Angle of Departure (AoD), or Time of Flight (ToF). As shown in Figure 1, each propagation path is received with a specific AoA and AoD, which introduce a corresponding phase shift across the antenna array due to extra travel distance. The introduced phase shift of $n^{t h}$ path with AoA of $\theta_{n}$ at $q^{t h}$ receiving antenna can be expressed as

$$
\Phi\left(\theta_{n}\right)=e^{-j 2 \pi f d \sin \left(\theta_{n}\right) / c}
$$

where $d$ is the distance between antennas. Similarly, the signal emitted from a linear transmit array will be received with a phase shift of $\Psi(\varphi)$, which is a function of AoD:

$$
\Psi\left(\varphi_{n}\right)=e^{-j 2 \pi f d^{\prime} \sin \left(\varphi_{n}\right) / c}
$$



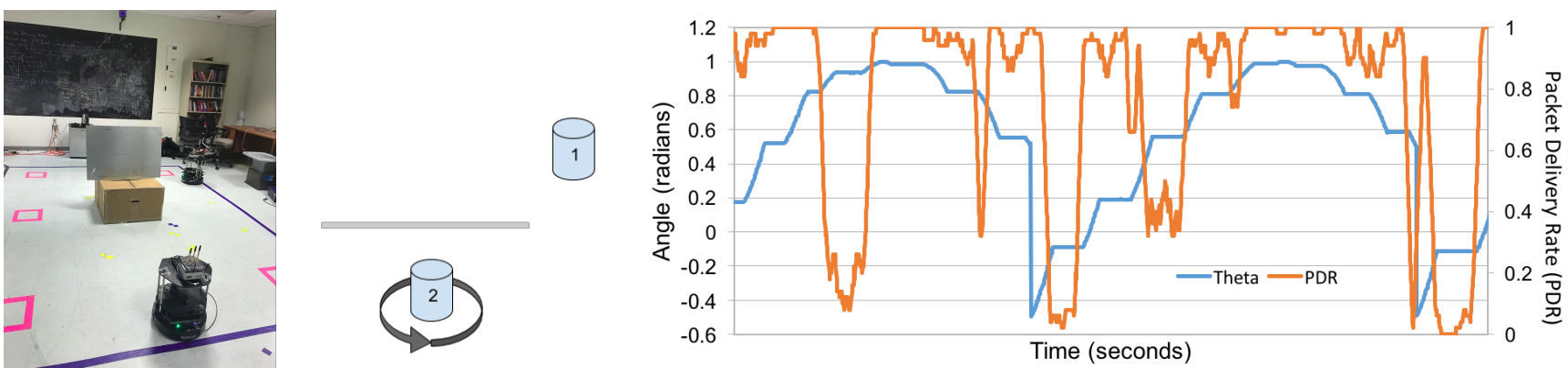

Figure 3: A small change in path lengths caused by the change of antenna array's orientation can alter the wireless channel quality from good to bad.

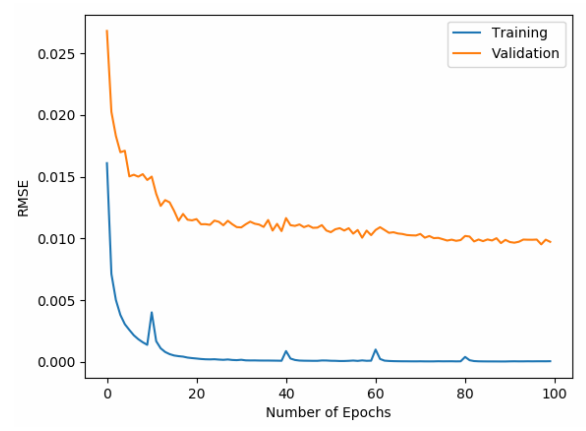

(a)

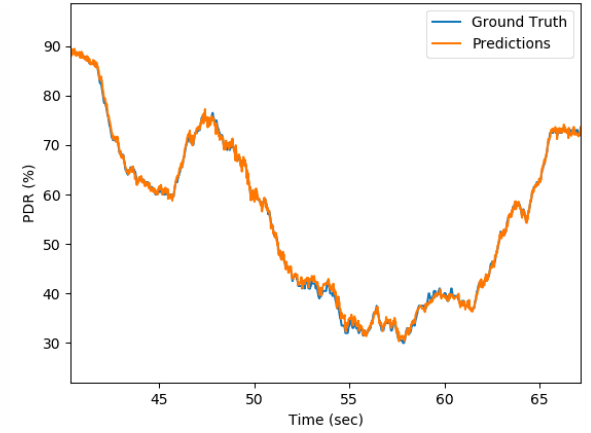

(b)

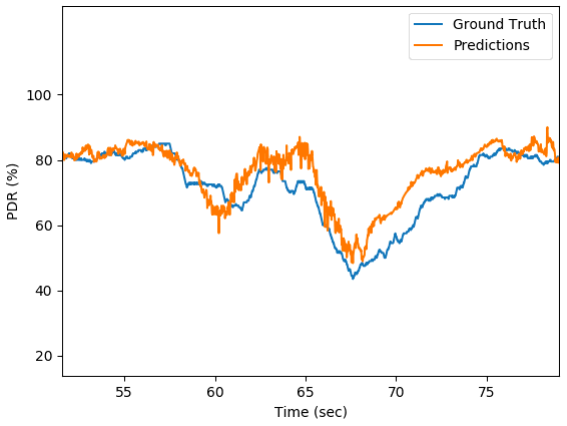

(c)

Figure 4: (a) Convergence of the training and validation loss function, (b) A snapshot from the training data and (c) the validation data, showing the ability of the trained LSTM model to predict PDR with $98 \%$ and $91 \%$ accuracy, respectively.

where $d^{\prime}$ is the distance between transmitting antennas. Therefore, with multiple antennas at the transmitter or receiver or both, we can distinguish the direction of the incoming signal [5]. Figure 3 demonstrates the empirical results from an experiment in which one node is static and the other is rotating, changing the multipath directions. We also added a metal plate as a synthetic reflector to reinforce the importance of multipath directions in causing destructive interference and accordingly packet loss. We can clearly see the drop of the packet delivery rate at certain orientations, while the distance between the two nodes remain the same across the entire experiment.

We use the spatial and frequential diversity in CSI measurements to characterize multipath propagation, and leverage temporal correlation of CSI measurements as well as their correlation with the partial model of the physical environment to assess the channel quality and predict the packet delivery rate. We implement this method with a Long Short-term Memory (LSTM) network that captures a sequence of PHY layer CSI in a time window, the physical information of the vehicle, and the predicted future trajectory states of the vehicle over a time horizon. We define packet delivery rate in the time horizon as the output of the LSTM by using a fully connected network in the last layer.

\section{PRELIMINARY RESULTS}

To evaluate the performance of the prediction method, we set up two turtle bots equipped with Intel 5300 cards, 3 antennas, and CSI tool [3] to capture channel state information. To create a dynamic indoor environment that imposes fluctuations in the wireless channel quality, we added $30 \mathrm{~d} b$ attenuators to each antenna, thus greatly reducing the transmission power relative to the testbed area. Next, we moved the two turtlebots in random motions around the test area using a transmission frequency of $50 \mathrm{~Hz}$ over a time horizon of $T=1 \mathrm{~s}$. We record a dataset of 24,000 data samples and used $80 \%$ for training and the rest for validation. As shown in Figure 4, the trained LSTM network achieved a 98\% prediction accuracy in training and a $91 \%$ accuracy in validation. In our future work, we demonstrate how vehicle controllers can use the predicted PDR values to not only preserve safety, but also optimize for a better wireless connectivity, resulting in an efficient motion planning.

\section{REFERENCES}

[1] N. Baccour, A. Koubâa, L. Mottola, M. A. Zúñiga, H. Youssef, C. A. Boano, and M. Alves. Radio link quality estimation in wireless sensor networks: A survey. ACM Transactions on Sensor Networks (TOSN), 8(4):34, 2012.

[2] R. R. da Silva and H. Lin. Safety Certified Cooperative Adaptive Cruise Control under Unreliable Inter-vehicle Communications. 2016.

[3] D. Halperin, W. Hu, A. Sheth, and D. Wetherall. Predictable 802.11 packet delivery from wireless channel measurements. ACM SIGCOMM Computer Communication Review, 41(4), 2011.

[4] C. Nowzari and J. Cortés. Distributed event-triggered coordination for average consensus on weight-balanced digraphs. Automatica, 68, 2016

[5] E. Soltanaghaei, A. Kalyanaraman, and K. Whitehouse. Multipath triangulation: Decimeter-level wifi localization and orientation with a single unaided receiver. In Proceedings of the 16th Annual International Conference on Mobile Systems, Applications, and Services, pages 376-388. ACM, 2018.

[6] J. Zhao and R. Govindan. Understanding packet delivery performance in dense wireless sensor networks. In Proceedings of the 1st international conference on Embedded networked sensor systems, pages 1-13. ACM, 2003. 